\title{
Le diplôme d'études spécialisées de réanimation
}

\author{
Critical care medicine as a primary speciality
}

\author{
J. Dellamonica $\cdot$ J.-L. Diehl $\cdot$ S. Dray $\cdot$ M. Fartoukh $\cdot$ P. Ferreira $\cdot$ P. Hubert $\cdot$ P.-F. Laterre $\cdot$ B. Levy $\cdot$ J.-P. Mira $\cdot$ \\ B. Misset $\cdot$ X. Monnet $\cdot$ H. Outin $\cdot$ J. Pugin $\cdot$ F. Tamion $\cdot$ pour le conseil d'administration de la Société de réanimation \\ de langue française (SRLF)
}

(C) SRLF et Springer-Verlag France 2014

\section{« Colère et intolérance sont les ennemis d'une bonne compréhension.» \\ Gandhi}

Début décembre 2013, nous avons eu la surprise de découvrir sur le site d'une société savante amie, la Société française d'anesthésie réanimation (Sfar) (www.sfar.org), un éditorial qui clairement s'oppose à la création du diplôme d'études spécialisées de réanimation en utilisant un argumentaire « discutable », rempli d'imprécisions et de contre-vérités. En particulier, les auteurs anonymes de ce texte entrevoient dans la création de ce DES de réanimation, un risque pour l'anesthé sie-réanimation de perdre sa compétence en réanimation! Cette peur est d'autant plus étonnante et incompréhensible pour les réanimateurs (médicaux) que lors de toutes les discussions sur ce sujet aux ministères et avec la Commission Nationale de l'Internat et du Post-Internat (CNIPI), les représentants de nos collègues anesthésistes-réanimateurs ont toujours été présents et que, bien entendu, il n'a jamais été envisagé une seule fois que l'anesthésie-réanimation puisse devenir l'anesthésie quand le DES de réanimation sera créé ! Mi-décembre sur le site du Syndicat national des praticiens hospitaliers anesthé sistes-réanimateurs (SNPHAR) (http://www.snphar.com/), un éditorial similaire agitait également le risque d'une anesthésie amputée de la réanimation et menaçait les pouvoirs publics d'actions si le DES de réanimation était créé ! Comment en sommes-nous arrivés à ce niveau d'incompréhension entre deux sociétés amies? Absence de communication? Sûrement ! Désinformation ? On n'ose le penser. Manipulation ? À qui profiterait le blocage du dossier ? Sûrement pas à nos plus jeunes collègues, qui regardent ahuris ces joutes politiques tellement éloignées de leur pratique quotidienne. Peu importe. Il est temps de reprendre la discussion et que chacun s'explique au grand jour. C'est pourquoi le Conseil d'administration de la Société de réanimation de langue française (SRLF) a décidé de publier un communiqué des responsables du Collège national des enseignants de réanimation (CNER) et de la section 48.02 du Collège National des Universités (CNU) qui est une réponse « technique » à ces éditoriaux [1].
En complément de ce texte pédagogique, nous voudrions insister sur trois points essentiels :

- la dimension européenne de la réanimation est bien connue de la SRLF. Avec un vice-président suisse et très prochainement un président belge (Dr Pierre-François Laterre), la SRLF a depuis sa création démontré une ouverture forte vers l'Europe, non seulement au niveau de son conseil d'administration mais à son plus haut niveau de responsabilités. Cette dimension européenne de la formation de réanimateur, nous avons également souhaité l'apporter grâce à l'internationalisation de notre congrès national et à travers les journées internationales de juin et notre revue Annals of Intensive Care ;

- la complémentarité des formations que réanimateurs et anesthésistes-réanimateurs proposent a toujours été reconnue par chacun, et il est surprenant que cela soit remis en cause. Les anesthésistes-réanimateurs ont ainsi introduit il y a quelques années dans leur cursus de formation la nécessité d'effectuer un an dans des services de réanimation (médicale). Le diplôme d'études spécialisées complémentaires (DESC) de réanimation contient un module de traumatologie et de réanimation périopératoires, qui est bien entendu majoritairement enseigné par des anesthé sistes-réanimateurs. Et surtout, en dehors des « niches » des services universitaires très spécialisés, l'immense majorité des services de réanimation français sont « mixtes » et y travaillent ensemble, pour le plus grand bien des patients, des anesthésistes-réanimateurs et des réanimateurs. Alors, pourquoi cette double formation devraitelle disparaître ? Qui bénéficierait de cet appauvrissement?

La filiarisation des formations spécialisées des épreuves classantes nationales 2010 est un fait politique irréversible. La fin des DESC en sera une des conséquences ou plus probablement a toujours été sous-entendue dans cette décision. Quel choix pour la réanimation ? Comme parfaitement expliqué dans le texte suivant, elle souhaite continuer à 
exister à côté de l'anesthésie-réanimation, dans un respect mutuel, une collaboration scientifique et médicale qui nous l'espérons continuera à faire des réanimateurs français les leaders européens de ces spécialités.

" Nous devons apprendre à vivre ensemble comme des frères, sinon nous allons mourir tous ensemble comme des idiots. ")

Martin Luther King
Conflit d'intérêt : le conseil d'administration de la SRLF déclare ne pas avoir de conflit d'intérêt.

\section{Référence}

1. Fourrier F, Vieillard-Baron A, Chastre J (2014) Le DES de réanimation : les vérités sont parfois bonnes à dire. Réanimation 23:1-2 